one critical bundle radius, derived from the relative energetic costs of filament bending and interfilament spacing distortion. Below this critical radius, the preferred morphology corresponds to bundles with a circular cross-section, whereas above it ribbon-like assemblies are favoured. Experimental and numerical verification of the authors' predictions provides reinforcement of this simple yet insightful theory.

Grason and colleagues' modelling study is an important step towards the quantitative understanding - and eventually, better control - of physical and chemical self-assembly. It defines the physical 'handles' with which the morphology of the assemblies can be tuned (it should be noted that the models are idealized and do not contain all the relevant elements; particularly, entropic and thermal effects are not yet included, and could turn out to be important). The task now is to find experimental ways of controlling and manipulating these physical parameters, for example via variation of temperature, solvents and concentrations.

Eran Sharon is at the Racah Institute of Physics, The Hebrew University of Jerusalem, Jerusalem, Israel. Hillel Aharoni is in the Department of Physics and Astronomy, University of Pennsylvania, Philadelphia, Pennsylvania 19104, USA. e-mail:erans@mail.huji.ac.il;

haharoni@sas.upenn.edu

\section{References}

1. King, H., Schroll, R. D., Davidovitch, B. \& Menon, N. Proc. Natl Acad. Sci. USA 109, 9716-9720 (2012).

2. Irvine, W. T. M., Vitelli, V. \& Chaikin, P. M. Nature 468, 947-951 (2010).

3. Hure, J., Roman, B. \& Bico, J. Phys. Rev. Lett. 106, 174301 (2011).

4. Meng, G., Paulose, J., Nelson, D. R. \& Manoharan, V. N. Science 343, 634-637 (2014)

5. Hall, D. M., Bruss, I. R., Barone, J. R. \& Grason, G. M. Nature Mater. 15, 727-732 (2016).

6. Chiti, F. \& Dobson, C. M. Annu. Rev. Biochem. 75, 333-366 (2006).

7. Seung, H. S. \& Nelson, D. R. Phys. Rev. A 38, 1005-1018 (1988).

8. Schneider, S. \& Gompper, G. Europhys. Lett. 70, 136-142 (2005).

9. Bruss, I. R. \& Grason, G. M. Proc. Natl Acad. Sci. USA 109, 10781-10786 (2012).

10. Bruss, I. R. \& Grason, G. M. Soft Matter 9, 8327-8345 (2013).

\title{
THIS SEASON'S COLOURS
}

When the microscope was first invented in the early seventeenth century there was a hope that it would reveal mechanical origins for all of nature's properties. That, alas, was not the case. But Robert Hooke discerned that colour, at least, could result from the "curious and exceeding smallness and fineness" of nature's parts. He studied the colours of bird plumage, pronouncing them "fantastical" because they would vanish when the feathers are wet. He correctly deduced that they arise "from the refractions of the light".

Coloration from light scattering was studied by Michael Faraday, David Brewster and John Tyndall in the nineteenth century, but only recently has it become feasible to fabricate nanostructures with the fineness and control needed to mimic nature's structural colours. The variety of these natural structures is remarkable, stemming from what looks for all the world like a kind of biological precision engineering ${ }^{2}$. Typically they consist of complex periodic arrays of scattering elements, such as rods or plates of melanin or chitin in feathers and butterfly wing scales. The ordered porous network known mathematically as a gyroid, found to be responsible for the bright green reflectivity of the Emeraldpatched Cattleheart (P. sesostris) and Green Hairstreak (C. rubi) butterflies, is perhaps the most elegant and ornate of them all'.

Turner et al. have previously used photopolymerization to reproduce the gyroid structure in a photoresist material, scaled up in size so as to work at infrared wavelengths ${ }^{4}$. Turner's group has now used optical lithography to write a gyroid network into a photo-resin with a lattice constant as small as $300 \mathrm{~nm}$, less than the C. rubi lattice of $350 \mathrm{~nm}$ and which reflects strongly in the near ultraviolet ${ }^{5}$.

One can imagine nature shrugging at this technical feat, reliant as it is on sophisticated photonic technology rather than the mild self-organization of organic membranes that produces the butterfly structures ${ }^{6}$. Yet, wet chemical methods are sufficient for making synthetic structural colour too - or nearly so. Galinski et al. have created a wide range of highly saturated colours, from yellow to blue, in composite thin films in which the light-scattering porous networks are made by selective dissolution of a metal alloy ${ }^{7}$. They remove the reactive aluminium from 300-nm-thick films of a platinum-yttrium-aluminium alloy, leaving a random porous network. To create strong reflectivity at a specific optical wavelength, the researchers deposit a dielectric film of alumina on top using sputtering, the colour being governed by the thickness of this layer (tens of $\mathrm{nm}$ ).

The colour-generating mechanism here is complex, without a known natural analogue. It involves an interaction between light reflected internally in the alumina film, which acts as a waveguide, and excitations called surface plasmon polaritons at the metal/dielectric interface. The

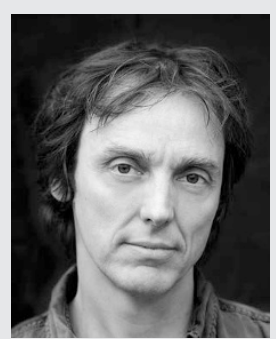

PHILIP BALL

convoluted nature of the interface induces a variation in refractive index, and the plasmons get trapped in nanoscale regions where this surface is convex. Resonant coupling associated with the waveguided modes within the alumina layer then allows some wavelengths to be strongly captured within the film while others are reflected.

The result is a material that is light and robust and which generates strong, tunable and non-iridescent colours - suitable, Galinski et al. say, for applications in the automobile or aeronautical industries, and perhaps for optical energy harvesting.

References

1. Hooke, R. Micrographia Observation XXXVI (J. Martyn \& J. Allestry, 1665).

2. Vukusic, P. \& Sambles, J. R. Nature 424, 852-855 (2003).

3. Michielsen, K. \& Stavenga, D. G. J. R. Soc. Interface 5, 85-94 (2008).

4. Turner, M. D., Schröder-Turk, G. E. \& Gu, M. Opt. Express 19, 10001-10008 (2011)

5. Gan, Z., Turner, M. D. \& Gu, M. Sci. Adv. 2, e1600084 (2016).

6. Saranathan, V. et al. Proc. Natl Acad. Sci. USA 107, 11676-11681 (2010).

7. Galinski, H. et al. Preprint at http://www.arxiv.org/ abs/1605.03700 (2016). 